Gehoben sind im Laufe des Monats

$1812000 \mathrm{~kg}$ um $20 \mathrm{~m}$, entsprechend $36240000 \mathrm{mkg}$ 450000, , $7 \mathrm{~m}$, " 3150000 , $68000,, \quad 6 \mathrm{~m}$, ", 408000,

insgesamt also eine Arbeit ge leistet von $49798000 \mathrm{mkg}$.

Demgegenüber ver b r a c h $t$ :

$3819 \mathrm{KW}$.-Stunden à $367000 \mathrm{mkg}=1402000000$ mkg. Der Nutzeffekt oder Wirkungsgrad beträgt demnach nur 3,5\%.

$\mathrm{Da}$ bei der zurzeit allgemein üblichen Art der Säurebeförderung innerhalb der Fabrik der Wirkungsgrad ein derartig ungünstiger ist, muß gewiß überraschen. Nimmt man an, daß $80 \%$ des Stroms vom Elektromotor in Kraft umgewandelt werden, und von dieser $50 \%$ im Kompressor verloren gehen, so bleibt noch ein Verlust von $40-3,5=36,5 \%$ nachzuweisen. Ein beträchtlicher Teil davon geht naturgemäß mit der komprimierten Luft verloren, die, nachdem sio ihrem $Z$ weck gedient hat, entweicht, ohne weiter ausgenutzt zu werden, und ein jedenfalls nicht geringerer durch die schon erwähnte Reibung in den Rohren, welche im vorliegenden Fall - $1150 \mathrm{l}$ in $8 \mathrm{Min}$. durch Rohre von $50 \mathrm{~mm}$ Durch. messer - von der Säure mit einer Geschwindigkeit von $1,4 \mathrm{~m}$ pro Sekunde durchströmt werden. Dazu kommen dann noch kleinere Verluste, wie der durch Reibung innerhalb der Transmission, durch Un- dichtheiten der Luftleitung oder -behälter usw., so daß es wohl erklärlich wird, wenn schließlich nur $3,5 \%$ von der angewandten Energiemenge übrig bleiben.

Es ist augenscheinlich, daß wir uns hier auf einem Gebiet befinden, auf welchem noch Ersparnisse zu erzielen sind; besonders ein Vergleich mit dem Kraftverbrauch für die Kühlwasserhebung läßt dies hoffen; die für diesen $\mathrm{Z}$ weck dienende mit Elektromotor gekuppelte Zentrifugalpumpe arbeitete nämlich in dem Messungsmonat mit einem Nutzeffekt von $30-32 \%$.

Sollten sich ähnliche Resultate auch bei der Säurehebung erreichen lassen, so wird auch die Fabrikationsweise nach $\mathbf{R}$ a s $c h$ ig s Vorschlag günstigere Chancen haben; vorläufig aber müssen wir mit den vorhandenen Verhältnissen rechnen, und da würde ein derartiges Schwefelsäuresystem sich wegen der überaus großen Kosten für Süurehebung schwerlich rentieren. R a schig spricht von Hebung des Vierzehn- bis Einundzwanżigfachen der Produktion; da doch auch der Wassergehalt der dünneren Säure noch zugeschlagen werden muß, so mag mal mit dem Einundzwanzigfachen des Produktionsgewichts gerechnet werden. Bleiben wir nun bei unserm oben vorgeführten Beispiel und berücksichtigen noch, daß die Druckhöhe entsprechend der zweckmäßig vergrößerten Höhe der Kammern sich auch vergrößert, vielleicht auf $22 \mathrm{~m}$, so würde die zu leistende Arbeit folgende sein:

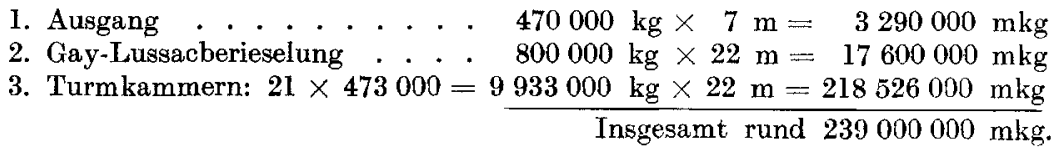

Dieselben entsprechen

$$
\frac{239000000 \times 100}{3,5 \times 367000}=18607 \mathrm{KW} \text {.-Stunden à } 7 \text { Pf. }
$$$$
=1302 \mathrm{M} 49 \mathrm{Pf}
$$

oder pro $100 \mathrm{~kg}$ produzierte Säure $50^{\circ}$ Bé. 27,5 Pf, d. h. $27,5-5,6=21,9$ Pf M e hr k o $\$$ t e n gegenüber der jetzigen Fabrikationsweise. Nicht berücksichtigt ist, daß die Säure auch noch mit etwa 3 Atm. Druck in die Düsen gelangen muß, die dafür nötige Kraft könnte wohl ausgeglichen werden (s. o.) durch die Vermeidung der Reibungskraftverluste in den Rohren, da die Säure langsam und gleichmäßig hochsteigt. Nun aber kommen noch hinzu die Kosten für Reparaturen und Beaufsichtigung der großen Hebungs- und Zerstäubungseinrichtung, für die Kühlung der Säure und für die vielen großen Säurereservoire, die viel wertvollen Platz wegnehmen. Es ist aber kaum wahrscheinlich, daß einem solchen Kostenaufwand überwiegende Vorteile des Verfahrens gegenüberstehen sollten, mögen solche nun in Vergrößerung der Leistung pro Raumeinheit bestehen oder in Salpeterersparnis; der ganze Salpeterverbrauch beträgt ja nur etwa 15 Pf pro $100 \mathrm{~kg} 50^{\circ} \mathrm{Bé}$.

Vielleicht aber - und insofern halte ich die von $R$ a s c hig gegebene Anregung für sehr beachtenswert - könnten mit einer beschränkten Einstäubung von dünner Säure, nämlich speziell in die letzté Kammer, Iohnende Ersparnisse an Salpeter erzielt werden, deren Geldwert größer wäre, als der für den vergrößerten $K$ raftbedarf, besonders, wenn es gelingt, den letzteren durch Vergrößerung des Wirkungsgrades der Hebungsapparatur zu verringern. Versuche, nach diesen beiden Richtungen hin eine Verbesserung und Verbilligung des Kammerprozesses zu suchen, könnten recht wohl von Erfolg sein.

\section{Zur Frage des Erfinderrechts der Angestellten.}

(Eingeg. 4./9. 1909.)

In Heft 36 dieser $Z$. bekämpft $O$. Wen $\mathrm{tzk}$ i die von mir (gelegentlich meiner Anregung der Schaffung eines Ausschusses für gewerblichen Rechtsschutz in unserem Verein) befürwortete Annahme der Stettiner Beschlüsse des ,Grünen Vereins" durch den Verein deutscher Chemiker. We n tzki stützt sich dabei auf die Anträge des Frankfurter Bezirksvereins, die auf unserer bevorstehenden Hauptversammlung zur Beratung kommen werden. In Anbetracht des letzteren Umstandes sehe ich hier von einer Besprechung der Anträge des Frankfurter Bezirksvereins ab, möchte aber einige Bemerkungen zu den Ausführungen We $n t z k$ is über die Besehlüsse des ,Grünen Vereins" machen, weil ich glaube, daß die Tragweite 
der Stettiner Beschlüsse von We ntzki und anderen doch nicht genügend gewürdigt wird.

Wentzki sagt, daß der ,Grüne Verein" die Angestelltenerfindung ,, tom rein rechtlichen Standpunkte behandelt und das sozialpolitisohe Moment dabei gänzlich ausgeschaltet habe." Dies ist jedoch durchaus nicht der Fall. Man hat in Stettin zuerst versucht; die wirtschaftliche Seite (im Grunde kann man wohl mit mehr Berechtigung neben der rechtlichen von einer wirtschaftlichen Seite als von einer sozialpolitischen Seite sprechen) von der rechtlichen in der Diskussion zu trennen, doch hat man dies bald aufgegeben, da eine strenge Durchführung dieser Trennung sich als unmöglich erwies; ich habe hierauf auch in meinem Bericht über den Stettiner Kongreß1) hingewiesen. Man hat also in Stettin sehr eingehend die vorliegenden wirtschaftlichen Verhältnisse geprüft und ist auf Grund dieser Prüfung teilweise einstimmig, teilweise mit überwältigender Mehrheit zu den bekannten Beschlüssen gekommen. Hierbei sei ausdrücklich bemerkt, daß eine große Anzahl von Juristen und Volkswirtschaftlern sich erst auf Grund der Debatte zu den oben genannten Beschlüssen bekannte.

Ich möchte ferner den ,Grünen Verein" und die Stettiner Verhandlungen insofern gegen We $\mathrm{ntzki}$ in Schutz nehmen, als sich der Punkt II, Satz 2, wie auch Punkt IVA. durchaus nicht gegen den Bund der technisch-industriellen Beamten allein richten. Fs sind vielmehr von verschiedenen Seiten früher ähnliche Forderungen und Behauptungen aufgestellt worden, wenn auch nicht ganz so weitgehend, wie der Bund technisch-industrieller Beamten sie jetzt vertritt, und gegen alle diese Ansichten richten sich die Punkte der Stettiner Beschlüsse. Namentlich Punkt IVA., der auf Veranlassung von Rechtsanwalt Dr. Is a y, Berlin, angenommen wurde, brachte zum Ausdruck, wie die Teilnehmer des Kongresses mit verschwindenden Ausnahmen über die geforderte Beschränkung der Vertragsfreiheit in unserem industriellen Leben denken.

Ich will nicht weiter auf die Ausführungen W e $n t z k$ is im einzelnen eingehen, wir werden $j a$ in Frankfurt noch Gelegenheit haben, darüber zu sprechen, möchte aber nicht unterlassen, darauf hinzuweisen, daß meines Erachtens die Frage der Angestelltenerfindung sehr wohl mit dem Patentgesetz in Verbindung gebracht werden kann, insofern, als das Patentgesetz schon jetzt verschiedene Bestimmungen enthält, die sich auf wirtschaftliche Verhältnisse beziehen, soweit sie sich auf Patentrechte gründen. Ich wiederhole hier, was Dr. W e n d t I a n d, der Vertreter des Bundes der Industriellen, in Stettin hervorhob, daß die vorliegende Frage zweifellos eine rein $v$ ol kswirtschaftliche ist, daß man sie erst zu einer sozialen stigmatisiert hat.

Bei dieser Gelegenheit möchte ich auf einen Punkt hinweisen, der auch einmal in unserem Verein zur Sprache gebracht werden sollte. Bei den Verhandlungen in unserem Verein wird sehr häufig dieser oder jener Bes o hlu B eines Bezir ksvereins als Grundlage benutzt, von dem man

1) Diese Z. 22, 1094 (1909). dann also annimmt, daß er die Ansicht des größten Teiles der Mitglieder jenes Bezirksvereins wiederspiegelt. Jeder aber, der sich einmal an dem Leben eines Bezirksvereins beteiligt hat, weiß, wie solche Beschlüsse zustande kommen: Von dem Vorstande des Bezirksvereins werden die Einladungen mit. Tagesordnung satzungsgemäß erlassen, zu der Versammlung findet sich aber meistenteils nur ein kleiner Bruchteil der Mitglieder ein, und dieser kleine Bruchteil faßt dann den Beschluß. Ich weiß natürlich, daß es kaum anders möglich ist, zu einem Beschlusse eines Bezirksvereins zu gelangen, aber ich möchte ausdrücklich betonen, daß man bei so wichtigen Fragen wie der vorliegenden nicht vergessen soll, daB wahrscheinlich ein s e hr g r o B e r Teil der Mitglieder des Vereins deutscher Chemiker anderer Ansicht ist, als die Beschlüsse des betreffenden Bezirksvereins dartun. Es sei mir gestattet, auf ein Beispiel hinzuweisen: Utber die Frage der Privatangestelltenversicherung haben vor einiger Zeit zwei große Bezirksvereine unseres Vereins einen Beschluß gefaßt. Die Mitgliederzahl der beiden Bezirksvereine zusammengenommen ist rund 650, bei der Fassung des obigen Beschlusses waren etwa 30 Mitglieder anwesend, also etwa 4,7\%. Ist man danach eigentlich berechtigt, anzunehmen, daß der Beschluß eines Bezirksvereins die Ansicht des überwiegenden Teiles der Mitglieder widerspiegelt? Ich glaube: nein!

Auf Grund dieser Betrachtungen scheint es mir auch recht wünschenswert, daß die Vertreter der Bezirksvereine nicht auf die Hauptversammlung kommen mit genauen, scharf begrenzten Anweisungen, wie sie abstimmen sollen, mit ,gebundener Marschroute". Die Vertreter der Bezirksvereine haben doch meist nur eine beschränkte Anzahl der Mitglieder ihres Bezirksvereins gehört, sie haben damit auch nur einen Teil der bestehenden Ansichten gehört; es ist doch sehr leicht möglich, daß sie auf der Hauptversammlung im Laufe der Erörterung ganz andere Ansichten bekommen, als sie vorher im Rezirksverein gehört haben. In solchen Fällen $m u ß$ es dem Vertreter des Bezirksvereins unbedingt freistehen, zu stimmen, wie er es auf Grund der Diskussion nach bestem Wissen und Gewissen für angebracht hält, nicht aber, wie es vielleicht der Bezirksverein ihm vorgeschrieben hat. Ich möchte diesen Punkt zur öffentlichen Diskussion stellen; vielleicht ist es angebracht, einen entsprechenden Passus in unsere Vereinssatzungen aufzunehmen.

L. M a $\times$ Wohlgemuth, Essen-Ruhr.

\section{Die Vorbildung der Chemiker.}

Das Folgende ist eine Fortsetzung und Ergänzung der nach Vorgang von C. D u is ber $\mathbf{g}$ von O. K a s e lit z über die Vorbildung der Chemiker aufgestellten Statistik (diese Z. 21, 5 [1908]). Zu den auf Tafel I und Tafel II (S. 1846) vorgeführten Ergebnissen ist zu bemerken:

Die Zahl derjenigen Chemiestudierenden, die das Verbandsexamen gemacht haben, ist im Laufe der Jahre von 1899 bis 1908 etwa dieselbe geblieben. Die Zahl der Ausländer bleibt von 1898 bis 1903 